\title{
Screening digital relay data to detect power network fault response anomalies
}

\author{
D. W. Kosy, V. S. Grinberg, and M. W. Siegel \\ The Robotics Institute, School of Computer Science \\ Carnegie Mellon University, Pittsburgh, Pennsylvania 15213-3891 USA
}

\begin{abstract}
An important part of power line protection system maintenance is the retrospective analysis of fault data to verify that all elements of the protection system were set properly and operated as they should have. In this paper, we describe an automated approach to detecting anomalies using data from microprocessor-based digital protective relays. At present, the systems are specific to the ABB Relay Division MDAR relay family. However, the techniques used are generalizable to other types and brands of digital relay.
\end{abstract}

\section{INTRODUCTION}

\subsection{Background}

Power transmission networks are protected from the damage that would result from prolonged short circuits in the network by a complex, interconnected collection of circuit breakers, protective relays, instrument transformers, and communications subsystems. These devices, together with some data recording equipment, comprise the protection system for the network. Short circuits that arise are called faults.

The decision-making elements in a protection system are the protective relays. These are integrated sensing and switching devices that measure the currents and voltages at network nodes, detect fault conditions (such as arcing initiated by lightning), and take protective action by tripping (i.e. activating) circuit breakers. The decision to trip is controlled by a relay's settings, which specify the types of electrical disturbances that are to be classified as actionable faults. In some systems, relays may also be set to reclose tripped breakers, after a short time delay, to restore service quickly when the fault is only a transient condition.

\subsection{Fault Response Analysis}

Whenever a circuit breaker is automatically tripped, the tripping event and the disturbance which caused it must be reviewed by maintenance engineers to verify that the protection system operated correctly. "Correct operation" means both (1) that each component of the system responded as it was supposed to and (2) that the system as a whole provided the degree of speed, sensitivity, and selectivity of operation desired for this particular case. Among the questions that need to be answered are the following:

- What was the state of the protection system at the time of the disturbance? Were the relays connected and set correctly? What states arose during the fault condition? Were the states of different relays responding to the same fault mutually consistent?

-What was the health of the protection system? Did activated breakers actually open? Did they open as fast as they should have? Does the fault response record indicate that all other components are in good working order? What additional maintenance tests should be ordered?

- What was the effectiveness of the protection system? Was response time adequate? Did relays reclose too soon? Does the fault record indicate that improved protection could be provided by changing settings, replacing components, adding components?

Since a protection system is designed to operate only during a fault, the occurrence of a disturbance provides the only opportunity to fully evaluate the system under actual field conditions.

Fault response analysis (also called "postmortem fault analysis" and "relaying performance analysis"1) is different from the kind of fault analysis performed by power station operators at the time of the fault. ${ }^{2-5}$ The purpose of that analysis is to decide whether the transmission network itself needs to be repaired or modified in some way in order to recover from a given fault. The purpose of fault response analysis is to decide whether the system protecting the network must be repaired or modified in some way to be able to respond effectively to future faults. 


\subsection{Digital Relay Data}

Traditionally, the primary inputs to fault response analysis have come from relay "targets" (visual indications of relay operations) and oscillograms (voltage, current, and control signal waveforms) both of which were recorded on paper at power substations. More recently, however, relays have been designed with sensing contacts through which the relay's switching state can be queried remotely via communication channels. The most modern relays incorporate local microprocessors that record instantaneous current and voltage measurements, contact states, control signals, and setting values as digital data. Via the communication channels, these data can be uploaded to personal computers where they can be displayed and manipulated by computer software. The next logical step, we believe, is to have the data analyzed by computer software as well.

\subsection{The MDAR Project}

In the summer of 1991, a project was begun under the sponsorship of the ABB Corporation to explore the use of expert system techniques for analyzing data recorded by MDAR digital relays, which ABB manufactures. Every MDAR has the capability to record:

- sixteen target values

- measurements of three-phase RMS currents and voltages, captured at the beginning of a disturbance

- calculated fault impedance and apparent distance to fault

for the 16 most recent disturbances seen by the relay. Some MDARs can also record, for each of these disturbances,

- three-phase current and voltage samples,

- 22 channels of binary data on the operation of internal relay logic,

- 2 channels on the receipt of external communication signals

for seven cycles after the disturbance was detected, and one cycle before, at 8 samples per cycle. Finally, every MDAR stores 62 setting values which configure it to a specific application.

Of primary concern initially was the size of the "mountain of data" that a collection of MDARs could produce as a thunderstorm swept through a power transmission network. The vast majority of tripping events in transmission networks are completely normal responses to transient faults caused by lightning strikes in the vicinity of transmission lines. But, within the hundreds of target values and thousands of measurements characterizing these events, there might also be indications of improper response. The concern was that an engineer could easily miss these clues, because the sheer volume of data would overwhelm the amount of time s/he could devote to analyzing it. Hence, it was felt that the first function an expert system should perform is a "screening" of the response data to flag any anomalies that might be present.

Also of concern was the problem of incorrect settings. No relay will respond properly if it has not been set correctly. As our work on response screening progressed, it became clear that we needed a settings checker to determine whether an anomalous response could be linked to an anomalous relay setting. To meet this need, we designed the checker described briefly in the next section. Following that, we describe the fault response screener in depth.

\section{SETTINGS CHECKER}

Figure 1 shows the parameters that must be set for an MDAR relay. The YES/NO/BLK/OUT values serve to activate/deactivate various options (e.g. whether or not the relay is to function in pilot mode, PLT), and the rest supply symbolic or numeric parameters (e.g., current transformer ratio, CTR).

The checker for these values is intended to alert maintenance engineers to potentially incorrect settings by flagging those that are abnormal. A "normal" setting value is one that is consistent with standard practice (e.g., a recommendation in the MDAR manual) for a standard transmission line in an unproblematic environment. Hence, an abnormal setting will be the result of either a nonstandard practice, a nonstandard line, a problematic environment, or a mistake.

The checker is mainly concerned with mistakes, i.e., cases where desired relay behavior was incorrectly communicated to the relay. There are many potential sources of error. A value may have been entered incorrectly. The person responsible for a setting may not have adequately understand some idiosyncrasy of the relay. A derived parameter may have been calculated incorrectly. The transmission system may have been changed in such a way that a change in relay settings is required, but was not made. The checker includes tests for all these different possibilities.

More interesting are cases where a setting is abnormal because it is unusual rather than erroneous. Since an abnormal setting may in fact be correct for a particular line, environment, and protection philosophy, the purpose of the checker is basically to request an engineer to document the reason an unusual setting value was chosen, rather than to critique that value or recommend an alternative. 


\begin{tabular}{|c|c|c|c|c|c|c|c|c|c|c|}
\hline OSC & dVdI & & 3TRM & NO & & ZR & $=3.30$ & & LOIB $=$ & $=\mathrm{NO}$ \\
\hline FDAT & TRIP & & BLKT & $=$ & msec & LV & 40 & VOLTS & AL2S & $=\mathrm{NO}$ \\
\hline CTR & 1000 & & RBSW & YES & & IL & $=0.50$ & AMPS & & \\
\hline VTR & 4000 & & PLTP & $=12.38$ & OHMS & IOS & $=0.50$ & AMPS & & \\
\hline FREQ & 60 & $\mathrm{~Hz}$ & PLTG & $=12.38$ & OHMS & IOM & $=1.00$ & AMPS & & \\
\hline CTYP & $=$ & AMP & z1P & 3.00 & OHMS & ITP & $=$ OUT & AMPS & & \\
\hline RP & NO & PRI & z1G & 3.00 & OHMS & ITG & OUT & AMPS & & \\
\hline XPUD & $=0.800$ & /DTY & T1 & No & & OSB & $=$ YES & & & \\
\hline DTYP & $=$ MILES & & $\mathrm{Z} 2 \mathrm{P}$ & $=12.38$ & OHMS & OSOT & $=4000$ & msec & & \\
\hline TTYP & OFF & & $T 2 P$ & BLK & SEC & $\mathbf{R T}$ & $=15.00$ & OHMS & & \\
\hline $62 T$ & $=5.00$ & SEC & Z2G & $=12.38$ & OHMS & RU & $=15.00$ & OHMS & & \\
\hline Z1RI & $=\quad \mathrm{NO}$ & & T2G & BLK & SEC & DIRU & $=$ ZSEQ & & & \\
\hline Z2RI & $=\mathrm{NO}$ & & z3P & $=12.38$ & OHMS & GBCV & $=$ OUT & & & \\
\hline Z3RI & NO & & T3P & BLK & SEC & GBPU & $=1.00$ & AMPS & & \\
\hline BFRB & $=\mathrm{NO}$ & & Z3G & $=12.38$ & OHMS & GTC & 12 & & & \\
\hline PLT & $=\mathrm{YES}$ & & T3G & BLK & SEC & GDIR & $=\mathrm{YES}$ & & & \\
\hline STYP & РОтT & & Z3FR & REV & & CIF & $=\mathrm{NO}$ & & & \\
\hline FDGT & $=$ & cycl & PANG & 80 & DEG. & LLT & $=\mathrm{NO}$ & & & \\
\hline WFEN & No & & GANG & 80 & DEG. & LOPB & $=$ YES & & & \\
\hline
\end{tabular}

Figure 1: Sample MDAR Settings

For example, it is standard practice to set the zone 1 reach of a distance relay to $70-90 \%$ of the protected line length. If set greater than this value, the relay may erroneously trip for faults beyond the far end of the line, due to unavoidable inaccuracy in establishing the location of a fault from measurements taken at the location of the relay. A relay engineer would say that the setting was not "selective" enough. If set less than this value, the relay will not trip for faults on the far end of the line which it could have recognized unambiguously. A relay engineer would say that the setting was not "sensitive" enough.

However, there are lines for which a different practice applies. This was the case for the settings in Figure 1. The setting checker output for the zone 1 setting values is shown below.

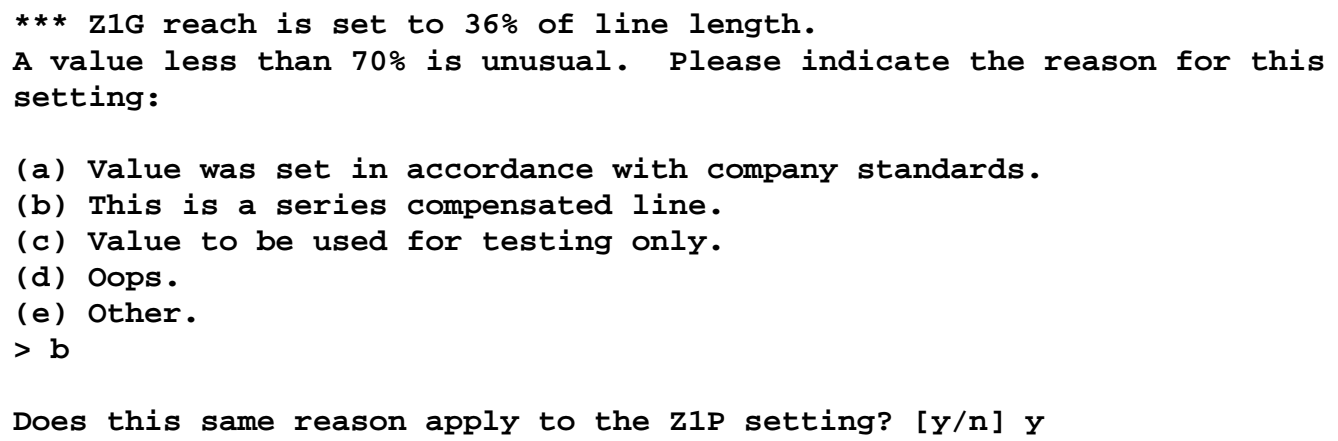

The final output of the checker is a report in which each setting value is annotated with one of the following evaluations:

- clerical error

- value does not match derivation from line parameters

- unusual but arguably correct setting

- unusual setting, not known to checker

- setting matches standard practice

- setting has no effect (due to other settings)

The last evaluation is supplemented with explanatory notes, such as the following:

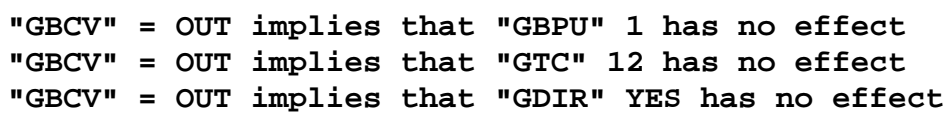

Due to time constraints, we included only enough tests in the checker to cover the settings practices for one particular relay installation. With more data from more installations, we expect that the number of "unusual settings, not known to checker" will decrease to zero. 


\section{FAULT RESPONSE SCREENING}

The response screening function is performed by a program called FRAN (for Fault Response ANalyzer). Figure 2 shows an example of an MDAR "target record". The values from "FTYP" to "DMI/DKM" reflect the relay's response to a disturbance. "PFLC", "PFLV", and "LP" are the prefault current, voltage, and power angle, respectively. The remaining values are measurements of the disturbance and derived symmetrical component values. Figure 3 shows several lines of MDAR oscillographic data. The labels across the top indicate the sources of the signals; e.g., "Ia" is phase A current, "Z1G" is the zone 1 ground pickup unit, "52b" is the breaker-open contact, "AG" indicates detection of a phase A to ground fault, etc.

\begin{tabular}{|c|c|c|c|c|c|c|c|c|c|c|c|c|}
\hline \multicolumn{5}{|c|}{ Sub :MPSI } & & 004 & MDAR TOP & \multicolumn{3}{|c|}{ (LEFT) } & & \\
\hline STATUS:C & & & & MDAR & FA & JLT (PREVI & US NO. & $1)$ & & & $1991 \quad 12$ & \\
\hline FTYP & $=$ & AG & TRIP & WFT & $=$ & NO & & v1 & MAG & $=$ & 52.84 & VOLTS \\
\hline BK1 & $=$ & YES & & ITP & $=$ & No & & & ANG & $=$ & 28.21 & DEG. \\
\hline BK1A & $=$ & YES & & ITG & $=$ & No & & v2 & MAG & $=$ & 13.35 & VOLTS \\
\hline BK1B & $=$ & YES & & CIF & $=$ & No & & & ANG & $=$ & -141.70 & DEG. \\
\hline BK1C & $=$ & YES & & LLT & $=$ & No & & $3 \mathrm{VO}$ & MAG & $=$ & 87.60 & VOLTS \\
\hline BK2 & $=$ & No & & GB & $=$ & No & & & ANG & $=$ & -145.00 & DEG. \\
\hline BK2A & $=$ & NO & & SPF & $=$ & No & & IPA & MAG & $=$ & 12.40 & AMPS \\
\hline BK2B & $=$ & NO & & $62 T$ & $=$ & No & & & ANG & $=$ & -53.00 & DEG. \\
\hline BK2C & $=$ & NO & & $\mathbf{z}$ & $=$ & 0.56 & OHMS & IPB & MAG & $=$ & 1.70 & AMPS \\
\hline Z1P & $=$ & NO & & FANG & $=$ & 49.00 & DEG. & & ANG & $=$ & 108.00 & DEG. \\
\hline z1G & $=$ & YES & DMI & /DKM & $=$ & 2.10 & MILES & IPC & MAG & $=$ & 1.30 & AMPS \\
\hline $\mathrm{Z2P}$ & $=$ & No & & PFLC & $=$ & 1.50 & AMPS & & ANG & $=$ & -10.00 & DEG. \\
\hline $\mathrm{Z} 2 \mathrm{G}$ & $=$ & No & & PFLV & $=$ & 67.20 & VOLTS & I1 & MAG & $=$ & 4.45 & AMP S \\
\hline Z3P & $=$ & NO & & LP & $=$ & -166.00 & DEG. & & ANG & $=$ & -65.71 & DEG. \\
\hline Z3G & $=$ & No & VPA & MAG & $=$ & 12.20 & VOLTS & I2 & MAG & $=$ & 4.18 & AMPS \\
\hline PLTP & $=$ & No & & ANG & $=$ & 0.00 & DEG. & & ANG & $=$ & -46.15 & DEG . \\
\hline PLTG & $=$ & No & VPB & MAG & $=$ & 75.60 & VOLTS & $3 I 0$ & MAG & $=$ & 11.83 & AMPS \\
\hline SEND & $=$ & YES & & ANG & $=$ & -100.00 & DEG. & & ANG & $=$ & -46.01 & DEG. \\
\hline $\mathrm{RX1}$ & $=$ & No & VPC & MAG & $=$ & 74.80 & VOLTS & IN & MAG & $=$ & 11.90 & AMPS \\
\hline $\mathrm{RX} 2$ & $=$ & NO & & ANG & $=$ & 161.00 & DEG. & & ANG & $=$ & -45.00 & DEG. \\
\hline
\end{tabular}

Figure 2: An MDAR Target Record

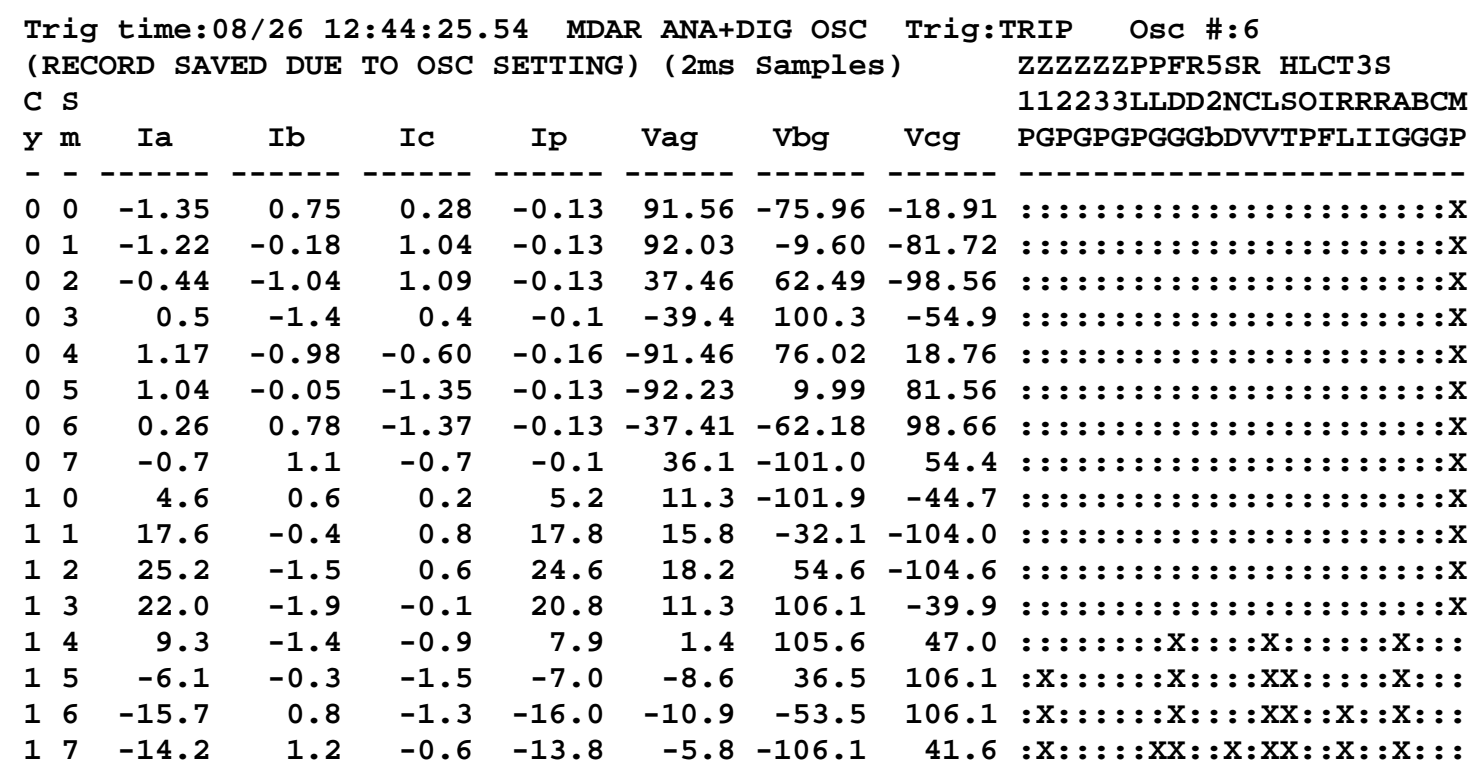

Figure 3: MDAR Oscillographic Information

The goal of screening is to determine from these data:

- A summary of what happened

- Any problems that are obvious

- Any inconsistencies that might be clues to more subtle problems 
To accomplish this, FRAN uses the reported fault type (FTYP), impedance (Z), and angle (FANG) to assemble a set of expectations about what should be observed in the rest of the response, and in the measurements, if this were a completely normal tripping event. The expectations are then tested against the data, by comparing values. Any significant differences are reported as abnormalities that should be investigated further.

The definition of "normal" also depends on two other types of input data: the settings and the network environment. For instance, if some element of the relay has been deactivated by a setting, one should not expect it to respond to any disturbance. With respect to environment, FRAN needs to know how many breakers a relay is connected to, the length and impedance of the protected line, the substations at each end of the line, the point of connection of the potential transformer, and where to find other records that may have come from a given disturbance. All target, oscillographic, settings, and environment records are stored in ordinary ASCII files in a hierarchical file directory structure.

\subsection{Screening the Response at One End of a Line}

The outcome of screening is best described by example. The following reproduces FRAN's output for the settings, target, and oscillographic records shown in Figures 1,2, and 3. User choices are underlined; clarifying comments are interspersed.

[Loading 08-26-91/1.sav for mpsl/004] [Osc is: mpsl/004/08-26-91/6.osc]

Target record 1.sav is one of the records recorded on August 26, 1991 for relay 004 at substation mpsl. The oscillographic record (6.osc) for an event does not necessarily have the same number as the target record. Correspondence is determined by comparing the time stamps of the records.

Searching for other /08-26-91 records of this disturbance. Found none.

For purposes of this example, data from the far end relay was temporarily removed from the directory structure.

Synopsis

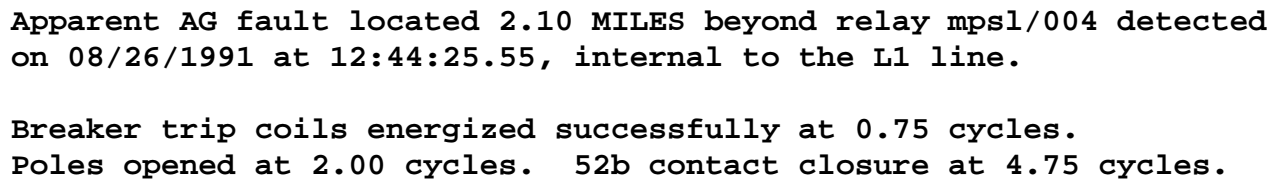

These lines summarize the disturbance the relay "thought" it saw (time, place, direction, phases involved, ground vs. non-ground fault) and present the key characteristics of the protective response (breaker operation, relay and breaker response times). Some of this information comes directly from the target record (cf. Figure 2), some of it is computed from the oscillographic record, and some is deduced from a combination of the settings, target and environment data. For instance, "breaker trip coils energized successfully ..." is concluded because: (1) the relay has the capability of single pole tripping but was set for three pole tripping (2) the relay was connected to one breaker, not two, (3) a YES value for BK1A, BK1B, and BK1C indicates that current flowed in each trip coil of the breaker.

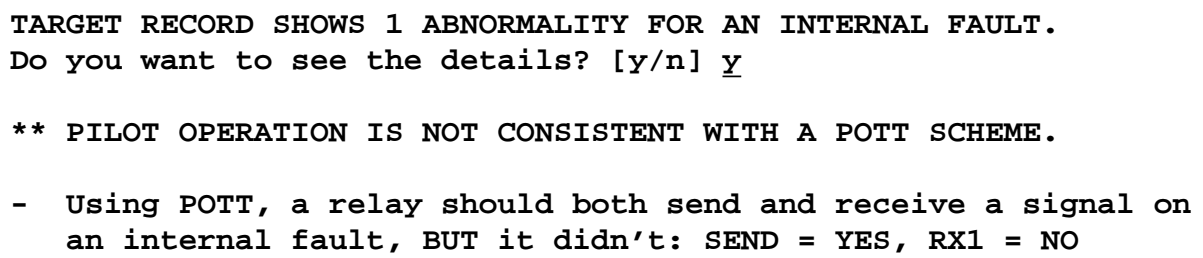

An MDAR configured for pilot operation can be set to use one of several pilot tripping schemes. This one was set to use permissive overreach transfer tripping (POTT), which requires receipt of a confirming signal from the far end relay before initiating a trip. It is abnormal not to receive such a signal with this scheme for a fault within (internal to) the protected line. 


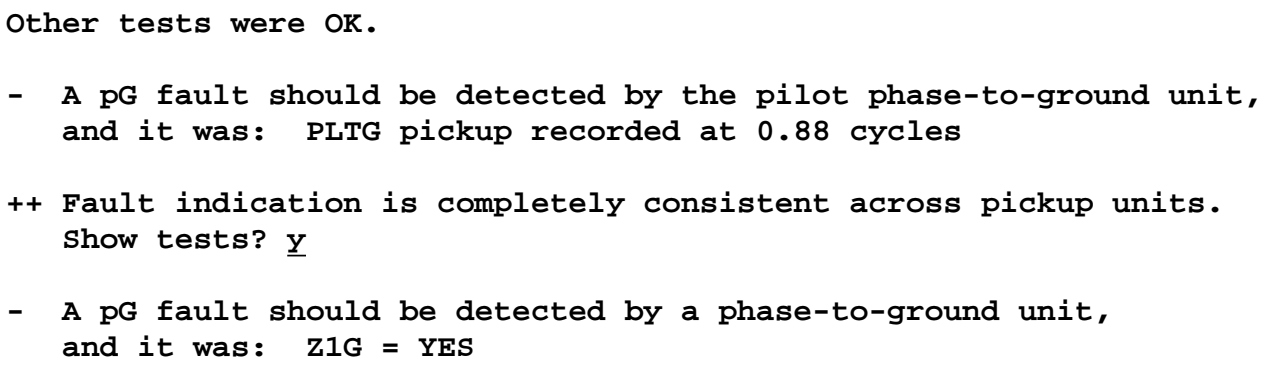

Tests are grouped into sections, with section evaluations marked "++" if all tests in that section were passed, and "**" if any were failed. The test results supporting a section evaluation are marked "-".

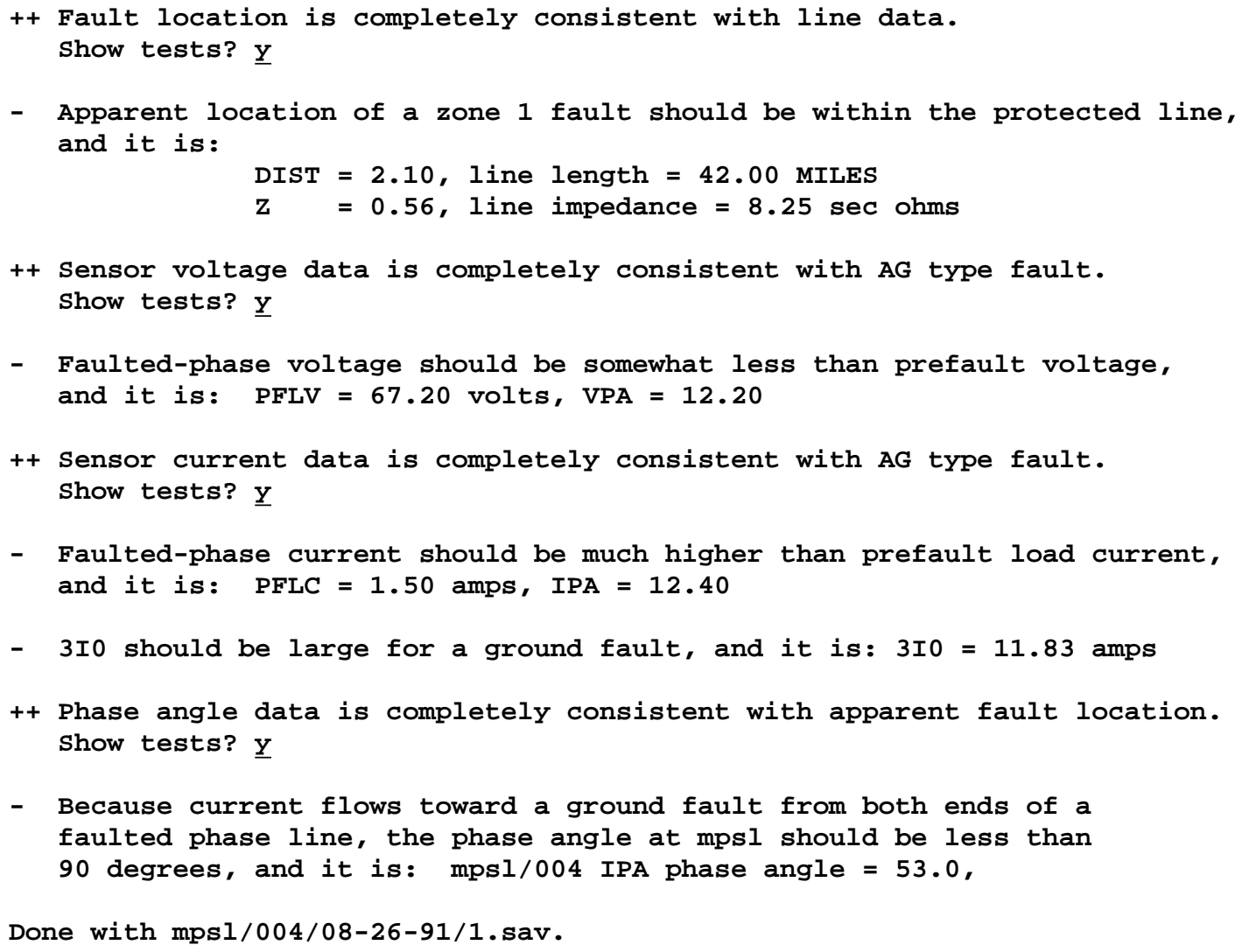

This example has displayed the majority of screening tests that FRAN uses, but not all of them. Different expectations are generated, and different tests applied, when a fault appears to be phase-to-phase, external vs internal, end-zone vs nearby, and so on. An important class of tests not shown are those that can be applied if data from the other end of the line are available.

\subsection{Screening with Data from the Other End of the Line}

Transmission lines are always protected by relays and breakers at both ends. If the far-end relay is an MDAR that generated a target record, FRAN can find it and use the additional information to provide a more accurate picture of the disturbance. In particular, two-ended data can be used (1) to determine conclusively whether a fault is internal or external to a protected line and (2) to compute a more accurate estimate of fault location. Schweitzer has shown how the latter can be used to identify previously unrecognized errors in line constants and relay reach settings. ${ }^{6}$ Comparing the data collected at one end with that collected at the other can yield other evidence of abnormality as well. 
The following output shows the effect of having target record information available from the far end relay for the case we have been analyzing. The various paragraphs would be interspersed in appropriate sections of the example output just presented.

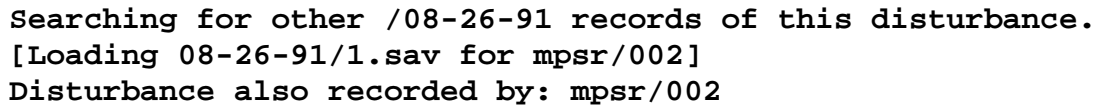

FRAN searches for records available from relays connected to the breaker(s) at the other end of the line, in the specified target group. Those that are close enough in time to the original record are read in for examination.

- The fault type seen by mpsl/004 should be the same as that seen by mpsr/002, and they agree: mpsl/004 FTYP = AG, mpsr/002 FTYP = AG

If there were a disagreement on fault type between the two ends, it would indicate either a relay problem, an evolving fault, or a fault that truly looks different from the different ends (e.g. a broken conductor whose two loose ends gyrate independently to create different short circuits). All of these conditions are abnormal and require further investigation.

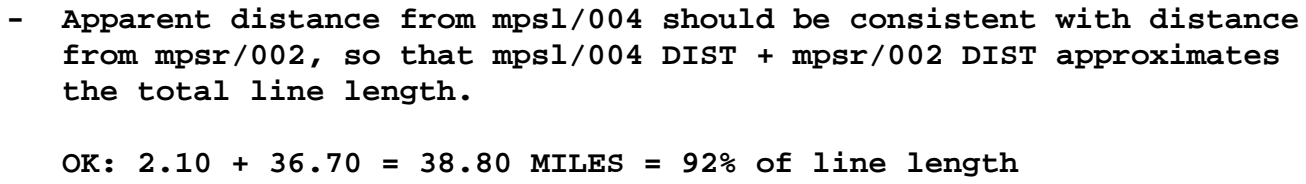

This test calls into question the line impedance values which are the basis for each relay's distance calculations. FRAN tolerates a $15 \%$ error in the sum. A two-ended calculation of fault location for this case later showed that a positive sequence angle of 72 degrees, rather than the 80 degrees to which the relays were set, yielded a sum that was almost exactly equal to total line length.

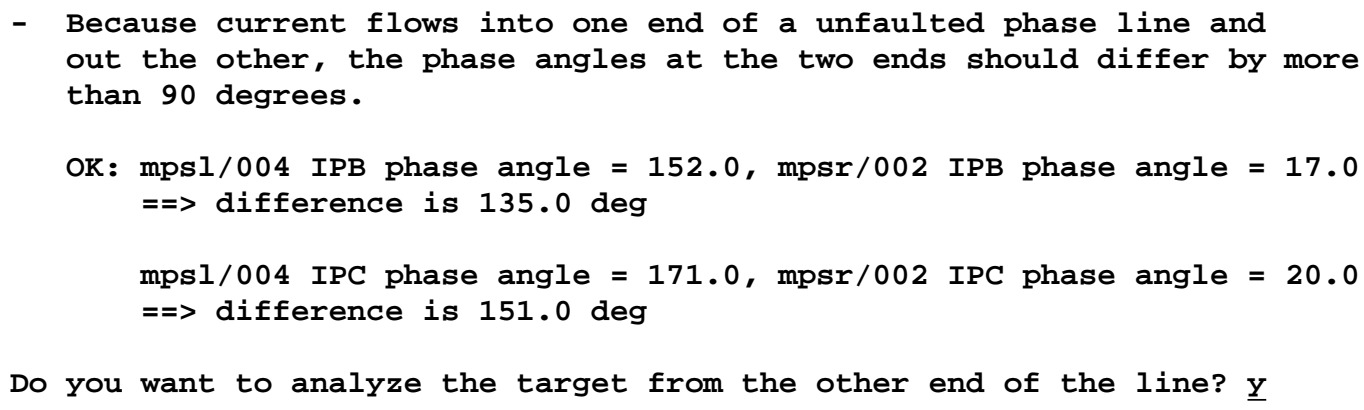

We definitely want to see the data from the other end because it may help us localize the source of the failure to receive a tripping signal, the only severe abnormality found by screening so far.

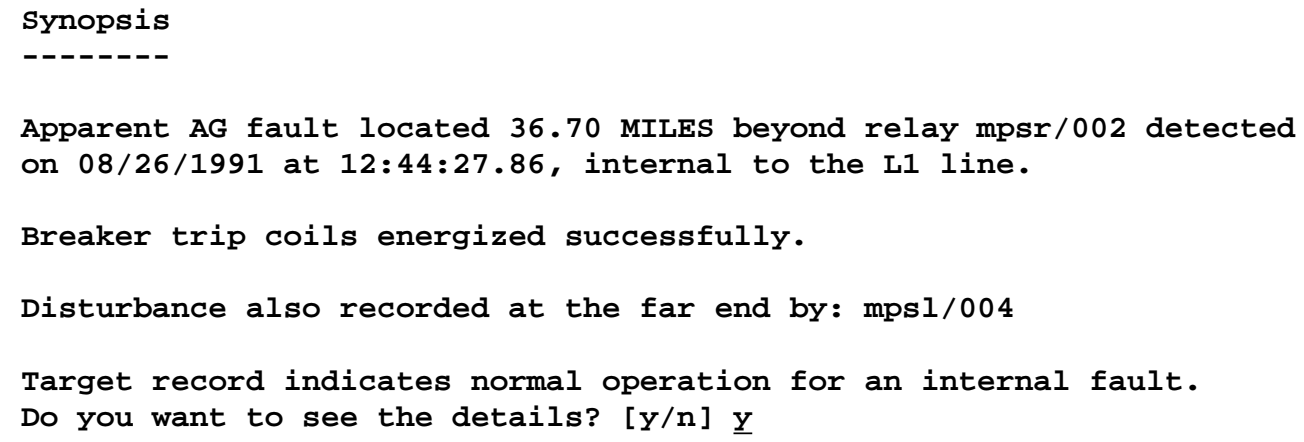

FRAN repeats the screening procedure for the other relay. Since it could find no oscillographic record, relay and breaker response times are not reported. The detail we want to see is pilot channel signals. 


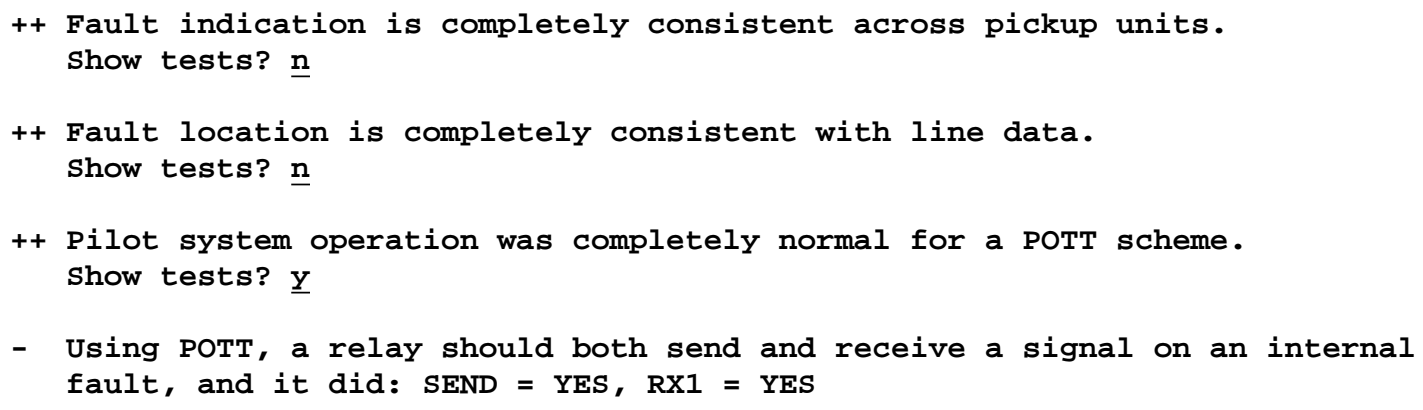

So, this end sent and received. The other end sent but did not receive. It would now be obvious to a maintenance engineer that the pilot communication channel is faulty.

\subsection{Misoperations}

A transmission line relay is said to "misoperate" when it trips for a disturbance not caused by a fault on the line(s) it is intended to protect. A large fraction of the tests exhibited in the previous section are designed to verify that a relay was not fooled into misoperating by unusual line conditions. In particular, any inconsistency between the voltage, current, or phase angle values expected for a given type of fault, and those actually observed, raises the possibility that the relay misoperated.

There are some cases in which the inconsistencies are so numerous and so substantial that FRAN can be certain that there was no line fault. The following is one such case:

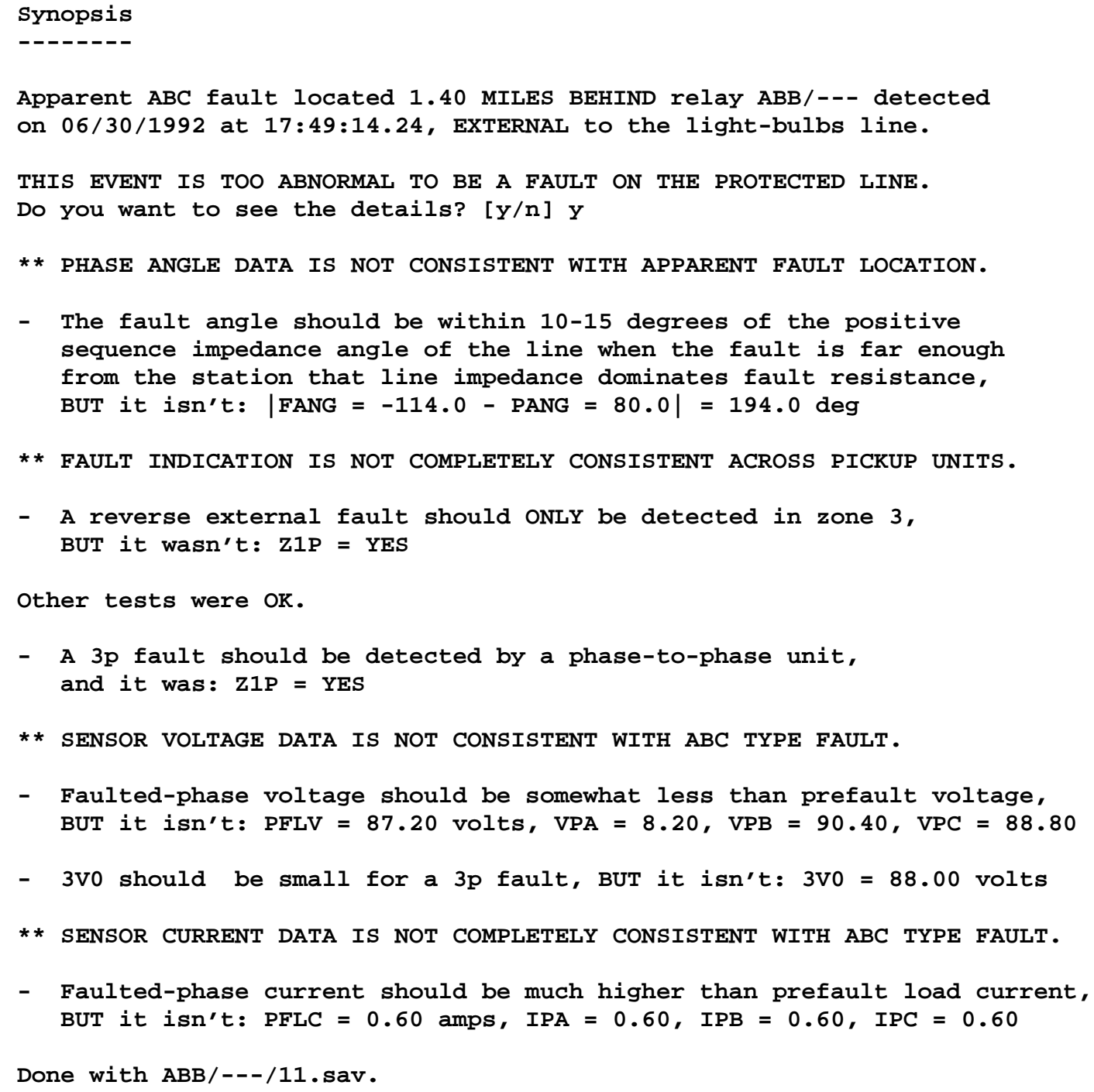


To classify an event as "too abnormal", FRAN keeps track of the number and severity of inconsistencies found. Too many small ones, or some small and some large, or any of several huge inconsistencies, will trigger the above sort of evaluation.

\subsection{Implementation}

Despite the apparent simplicity of the screening tests, the combination of settings, event, and environmental data required to perform them is surprisingly complex. This is so primarily because few tests are applicable under all circumstances, so each must be restricted to the situation in which it is relevant. For instance, the test that a relay successfully energized all circuit breaker trip coils is applicable only when:

- the relay is actually connected to a circuit breaker (environment)

- the target record says the relay decided to trip (event)

- the relay is set to trip all breaker poles (settings) or the fault involved more than one phase (event)

Similar multi-condition restrictions govern the application of most other tests.

FRAN is implemented as a forward-chaining rule-based system in the CLIPS language. ${ }^{7}$ The uses and flows of data within it are diagrammed in Figure 4. The readers convert external file formats into internal CLIPS format. Implication rules derive intermediate results such as percentage voltage drops and phase angle differences. Events are classified by location (internal vs external to line, near vs far from relay under analysis) and affected phases. The complexity of the applicability conditions is indicated by the number of inputs to the "comparison" box. The "tolerances and limits" represent engineering judgment as to how high or low a particular measurement must be to qualify as "large", "small", "much higher", etc. Rules for summarization, reporting and evaluation prepare various pieces of text for output.

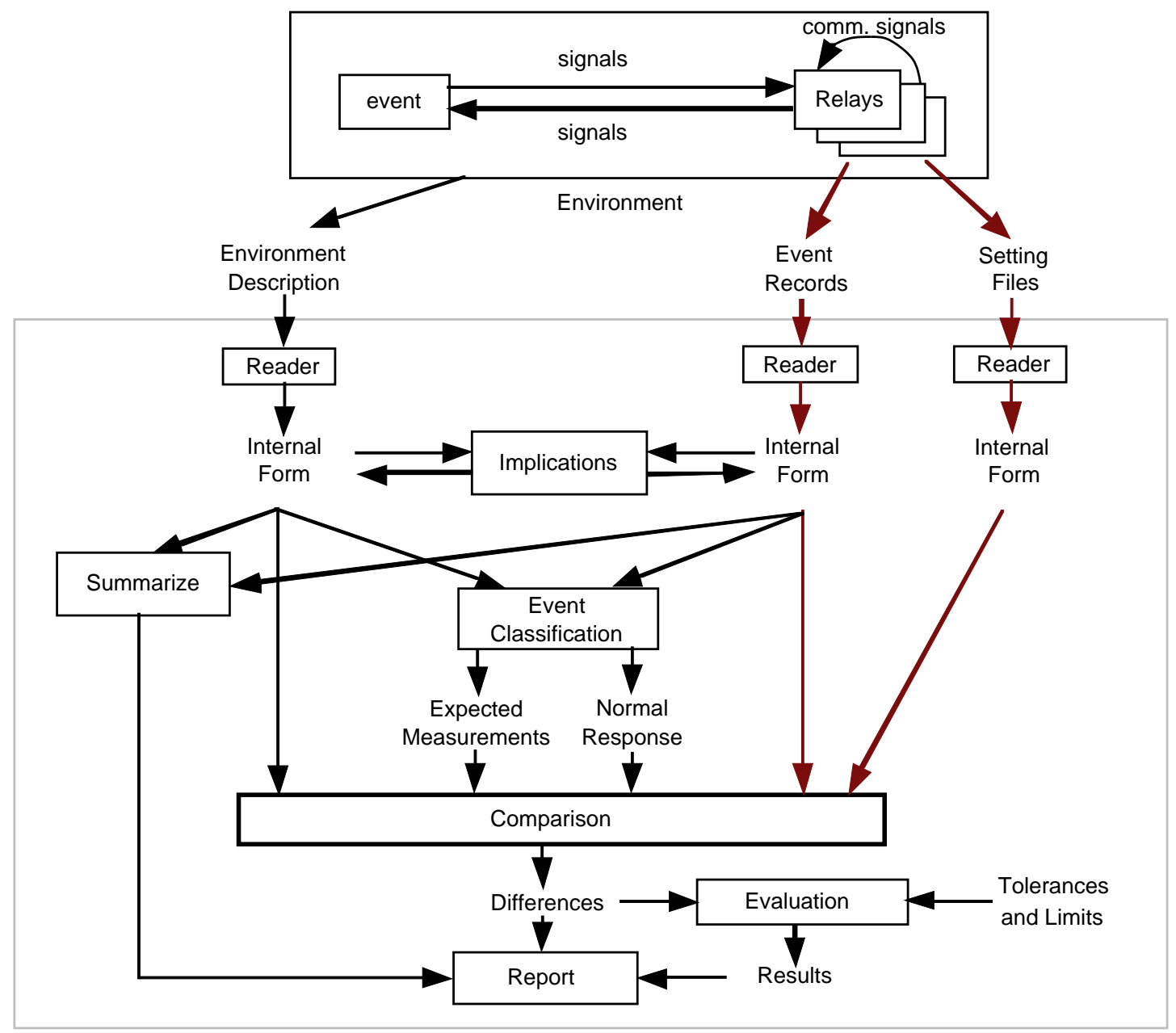

Figure 4: FRAN Data Flow 
CLIPS was chosen because:

- It runs on both IBM PCs and Unix-based workstations, and therefore, so does FRAN.

- It allows substantial sequential programming on the right-hand sides of rules (good for numerical algorithms and output formatting).

- Source code is distributed in $\mathrm{C}$ and the interpreter can be extended in, and called from, $\mathrm{C}$ programs. The readers, for example, are written in $\mathrm{C}$.

On either a PC or Unix platform, program response time is essentially instantaneous.

\subsection{Extensions}

At present, FRAN embodies 22 screening tests, all of which were suggested by consulting engineers at ABB as being particularly appropriate to MDAR data. There are many more that could be added which are relevant to other types of protection system elements. ${ }^{1,8,9} \mathrm{We}$ found the most comprehensive guide to general fault response analysis in the "Oscillograph Manual", produced by the Pennsylvania Electric Association. ${ }^{10}$ The backbone of this analysis is a set of 18 questions that an engineer should attempt to answer about a fault and a response. Of these questions, FRAN currently answers 9.*

There are basically two types of extensions required to answer the remaining 9 questions:

- Longer time window. FRAN currently considers only the data available in one 8-cycle MDAR record. Several of the questions require a longer perspective, e.g. "Were there any power swings after clearing?" "Were reclosing times appropriate?" One way to look at a longer interval than 8 cycles is to use more of the records that an MDAR can be set to produce.

- More oscillographic analysis. FRAN does not yet look at the shape of transient waveforms and hence does not test for breaker restrikes, prestrikes, instrument transformer distortion, or changes in fault type over time. Nor does it look at the prefault cycle for things like system imbalance prior to a fault. It does not check everything it could check regarding breaker and pilot channel operation.

Both of these are on our agenda for future work. However, we recognize that some questions may simply not be answerable with MDAR oscillography (or any typical digital relay oscillography) because of the limited time window and bandwidth of these data compared to data from standard fault recorders. More research is required to fully understand the limits on the use of relay data to do response screening.

\section{CONCLUSIONS}

Fault response analysis is an interpretation task in which the actual response of equipment to electrical stimuli is compared to expected or desired response patterns. Automating this task requires measurements of the stimuli, a record of the actual response, a computation of the response pattern expected or desired for those stimuli, and a detailed comparison from which evaluative conclusions may be drawn. While other types of equipment can record and report measurement data and contact-closure status over time, digital relay data is uniquely suited to automatic analysis because the goal-oriented nature of a relay's response can be used to derive the correct response pattern, in computational form, without any other information from the user. The results presented in this paper show that it is possible to capture enough of the comparison and evaluation criteria to automate at least half of the work of manual fault response analysis, if lines are protected by MDAR relays.

However, reduction of effort is not the only benefit provided by automatic analysis, nor even the most important. It can also be more thorough. The amount of time devoted to fault response analysis varies widely from one utility to another and from one disturbance to another. It is not uncommon for simple responses to be classified as "normal" if, on the surface, they indeed look normal. The example presented earlier might have been so classified, by the following logic: there was a storm; a line tripped out; the breakers operated at both ends; the line was reclosed and energized without further disturbance; looks OK. But, even though this particular tripping event was correct, there was a problem that will cause the system to malfunction in the future if certain types of faults arise. Since an analysis program will review every available aspect of a response on every occasion, it will always alert an engineer to hidden problems that might otherwise go unnoticed.

\footnotetext{
*The difference between 22 tests and 9 answers comes mainly from the fact that FRAN employs several tests to answer one question. For example, one of the questions is "Did the relays respond correctly given current and voltage signatures?" Almost half the tests are devoted to answering this one question." Besides that, FRAN answers a few questions the manual doesn't ask, primarily regarding cases of relay misoperation.
} 
We have not as yet demonstrated that analysis can also be automated for digital relays other than MDAR. However, it turns out that almost none of tests performed are conceptually restricted to MDARs. The parts of the programs that are MDARspecific have to do with the format of the input data, the presence of certain binary response values (e.g., whether breaker trip coils were energized), and the meaning to be ascribed to target indicators and setting values. Hence, adapting the programs to other types and brands of digital relay should require only writing new readers for their setting files and event records, and adding the appropriate target indicator tests.

\section{REFERENCES}

[1] "Pilot Relaying Performance Analysis", IEEE Power Systems Relay Committee Report, IEEE Transactions on Power Delivery, Vol. PWRD-5, No. 1, pp. 85-102, January 1990.

[2] P. Fauqembergue and L. Perrot, "An Expert System for the Fault Analysis of Electric Power Systems", Second Symposium on Expert Systems Application to Power Systems (Seattle, WA), pp. 83-90, July 1989.

[3] C. Fuku and J. Kawakami, "An Expert System for Fault Section Estimation Using Information from Protective Relays and Circuit Breakers", IEEE Transactions on Power Delivery, Vol. PWRD-1, No. 4, pp. 83-90, October 1991.

[4] M. Kezunovic, "Implementation Framework of an Expert System for Fault Analysis", Third Symposium on Expert System Applications to Power Systems (Tokyo-Kobe, Japan), pp. 80-86, April 1991.

[5] H. Sugihara, "Construction of Fault Analysis System for Power System", Third Symposium on Expert System Applications to Power Systems (Tokyo-Kobe, Japan), pp. 12-18, April 1991.

[6] E. O. Schweitzer "A Review of Impedance-Based Fault Locating Experience", presented at the Georgia Tech Protective Relaying Conference (Atlanta, GA), May 1989.

[7] CLIPS Reference Manual, Artificial Intelligence Section, Johnson Space Center, Houston, TX, April 1988.

[8] "Application and Evaluation of Automatic Fault-Recording Devices", IEEE Power Systems Relay Committee Report, IEEE Transactions on Power Apparatus and Systems, Vol. PAS-84, No. 12, pp. 1187-1203, December 1965.

[9] "Supplement to Application and Evaluation of Automatic Fault-Recording Devices", IEEE Power System Relay Committee Report, IEEE Transactions on Power Apparatus and Systems, Vol. PAS-90, No. 2, pp. 751-755, March/April 1971.

[10] "Oscillograph Manual", Pennsylvania Electric Association Relay Committee, Harrisburg, PA, December 1991. 